\title{
Variational Hierarchical Posterior Matching for mmWave Wireless Channels Online Learning
}

\author{
Nabil Akdim ${ }^{1}$, Carles Navarro Manchón ${ }^{2}$, Mustapha Benjillali ${ }^{3}$ and Pierre Duhamel ${ }^{4}$ \\ ${ }^{1}$ Apple, Munich, Germany \\ ${ }^{2}$ Department of Electronic Systems, Aalborg University, Denmark \\ ${ }^{3}$ Communication Systems Department, INPT, Rabat, Morocco \\ ${ }^{4}$ Laboratoire des Signaux et Systemes (L2S), CNRS-CentraleSup, France \\ Emails: nabil.akdim@apple.com, cnm@es.aau.dk, benjillali@ieee.org, pierre.duhamel@12s.centralesupelec.fr
}

\begin{abstract}
We propose a beam alignment algorithm that enables initial access establishment between two transceivers equipped with hybrid digital-analog antenna arrays operating in millimeter wave wireless channels. The proposed method builds upon an active channel learning method based on hierarchical posterior matching that was originally proposed for single-sided beam alignment on single path dominant channels. We extend it to the double-sided alignment problem and propose an estimation framework based on variational Bayesian inference that accounts for the uncertainties on the unknown channel complex gain and noise variance. The proposed approach is numerically shown to be resilient to the single path assumption and reaches near optimal beamforming gains with a moderate training overhead, even at low signal-to-noise ratios.
\end{abstract}

\section{INTRODUCTION}

Low power consumption and implementation complexity of hybrid digital-analog transceiver architectures have accelerated their adoption as a beamforming solution that can enable efficient wireless communications over the harsh mmWave frequency limited-scattering and blockage-prone wireless channels for $5 \mathrm{G}$ and beyond wireless cellular networks [1]. Also, angular sparsity of such channels [1], [2] allows for the use of adaptive sparsity-friendly techniques to ease the initial alignment and channel state information (CSI) acquisition on them, when using such transceiver designs [3]-[6].

In this study, we focus on hierarchical posterior matching (HiePM), an initial access scheme introduced in [7] which provably enables fast and reliable initial access establishment between two wireless transceivers over wireless mmWave channels with a single dominant path. Chiu et al. have shown in that contribution that using posterior matching [8] together with hierarchical beam search [4] can significantly reduce the initial access acquisition time while keeping the corresponding misalignment probability relatively low, provided that the channel's complex gain and operating signal-to-noise ration (SNR) are fully known to the communicating devices. These limiting constraints were relaxed in [9] by proposing to augment HiePM with extra simplifying assumptions on the statistical properties of the channel's CSI and then to use either a sampling scheme or a linear filtering scheme (Kalman filter) to learn it in parallel to running HiePM. Although this latter extension of the vanilla HiePM makes it robust with respect to uncertainties on the channel's CSI, it still presents some limitations. First, it assumes perfect knowledge of the operating SNR. Second, the assumptions made on the statistical distribution of the channel's complex gain (needed to make HiePM able to run as we will see later), are simplistic and not justified from a theoretical or practical view. Third, the proposed methods that build on such statistical assumption to overcome the CSI uncertainty issue are either very restrictive and computationally heavy (in the case of the sampling method) or show relatively moderate to low performance (in the case of the Kalman filter method). Finally, the overall tweaked setup assumes that one of the communicating transceivers has a single antenna, and the extension to the case where both communicating devices use the hybrid digital-analog transceiver structure is not straightforward.

Our contribution, in this work, which we dub "Variational HiePM (V-HiePM)" will address shortcomings of proposals in both of the aforementioned works [7], [9]. Specifically, we will augment HiePM with a variational approximate inference model [10] that will:

- make it robust against uncertainties of both CSI and operating SNR,

- allow for a natural and theoretically grounded parametrisation of the statistical properties of the CSI and operating SNR, in a way that will make HiePM run smoothly,

- make the overall setup performing very close to the vanilla HiePM scheme with perfect SNR/CSI knowledge,

- allow for both communicating devices to be equipped with hybrid digital-analog arrays.

\section{System ModeL}

Our system is composed of two hybrid digital-analog antenna array devices $A$ and $B$, equipped with uniform linear arrays (ULAs) of $N_{A}$ and $N_{B}$ antenna elements respectively. The elements on the ULAs are separated by a distance $d=\lambda / 2$, where $\lambda$ is the the mmWave wavelength of interest. Device $A$ ( $B$ respectively) digitally controls its ULA with $N_{A}^{R F}$ ( $N_{B}^{R F}$ respectively) RF chains. The two devices communicate over a reciprocal static and narrowband wireless mmWave MIMO channel, that is modeled as a $N_{B} \times N_{A}$ 
complex matrix $\boldsymbol{H}$, sampled from the finite scatterer channel model [5] with a single dominant path as ${ }^{1}$ :

$$
\boldsymbol{H}=\alpha \boldsymbol{a}_{B}\left(\phi_{B}\right) \boldsymbol{a}_{A}^{\mathrm{H}}\left(\phi_{A}\right)
$$

where $\alpha$ is the complex fading channel gain. $\boldsymbol{a}_{A}\left(\phi_{A}\right)$ and $\boldsymbol{a}_{B}\left(\phi_{B}\right)$ are the ULA array response vectors at devices $A$ and $B$ with incidence angles $\phi_{A}$ and $\phi_{B}$ respectively, modeled as $\boldsymbol{a}_{A}\left(\omega_{A}\right)=\left[1, e^{-j \omega_{A}}, \ldots, e^{-j\left(N_{A}-1\right) \omega_{A}}\right]^{\mathrm{T}}$ and $\boldsymbol{a}_{B}\left(\omega_{B}\right)=$ $\left[1, e^{-j \omega_{B}}, \ldots, e^{-j\left(N_{B}-1\right) \omega_{B}}\right]^{\mathrm{T}}$, with $\omega_{A}\left(\phi_{A}\right)=\frac{2 \pi}{\lambda} d \cos \left(\phi_{A}\right)$ and $\omega_{B}\left(\phi_{B}\right)=\frac{2 \pi}{\lambda} d \cos \left(\phi_{B}\right)$. The incidence angles $\phi_{A}$ and $\phi_{B}$ are assumed to be sampled from the ranges $\left[\theta_{A, 1}, \theta_{A, 2}\right]$ and $\left[\theta_{B, 1}, \theta_{B, 2}\right]$ respectively. ${ }^{2}$

The two devices go through an initial access phase consisting of a pilot based beam alignment procedure in order to establish the wireless link between them. We assume in this work that, during this initial access phase, the CSI learning and beam search processes for the two devices are centralized, i.e one of the devices, say $B$, is collecting measurements based on device $A$ 's pilot transmission, uses them to learn the channel's statistics and devises the beamformer it will use for the next pilot reception occasion together with the beamformer that device $A$ should use in sending that pilot, then communicates such information to device $A$ through an ideal, error-free control channel ${ }^{3}$. At time instant $t$, device $A$ sends a pilot symbol to $B$, which observes, after pilot removal:

$$
y_{B, t}=\sqrt{P} \boldsymbol{w}_{B, t}^{\mathrm{H}} \boldsymbol{H} \boldsymbol{f}_{A, t}+\boldsymbol{w}_{B, t}^{\mathrm{H}} \boldsymbol{n}_{B, t}
$$

where $\boldsymbol{f}_{A, t} \in \mathbb{C}^{N_{A}}$ and $\boldsymbol{w}_{B, t} \in \mathbb{C}^{N_{B}}$ are the effective beamformer and combiner used at time $t$ by transceivers $A$ and $B$ respectively. These are chosen from the hybrid digital-analog codebooks detailed next. $\boldsymbol{n}_{B, t} \in \mathbb{C}^{N_{B}}$ is a complex circularly-symmetric additive white Gaussian noise vector with i.i.d elements with an unknown variance $\sigma_{B}^{2}$, obtained after training sequence removal. $\sqrt{P}$ is the average transmit power of the pilot signal.

The adaptive beamforming strategy proposed herein utilizes the hierarchical beamforming codebook of [4]. Such a codebook, noted $\mathcal{C}_{S}$ hereafter, is designed to have $S$ levels of beam patterns. We note $\mathcal{C}_{l}$ the collection of beams belonging to level $l$. Then, $\mathcal{C}_{l}$ contains $2^{l}$ beamforming vectors that divide the sector $\left[\theta_{1}, \theta_{2}\right]$ into $2^{l}$ directions, each associated with a certain range of incidence angles $\mathcal{R}_{l}^{m}$, such that $\left[\theta_{1}, \theta_{2}\right]=\cup_{m=1}^{2^{l}} \mathcal{R}_{l}^{m}$. We note each of such $2^{l}$ vectors as either $\boldsymbol{f}_{A}\left(\mathcal{R}_{l}^{m}\right)$ or $\boldsymbol{w}_{B}\left(\mathcal{R}_{l}^{m}\right)$, depending on the considered device.

\section{Sequential Beam Pair Search via Variational HIERARCHICAL POSTERIOR MATCHING}

We start this section by first reviewing the details of the vanilla HiePM scheme [7], showing that knowledge of the

\footnotetext{
${ }^{1}$ This assumption is used only for the analytical derivation of our scheme. In Section IV, we show numerically that the method is resilient to such a limitation.

${ }^{2}$ No statistical assumptions on the distribution of the gain and the angles are made here, we will later justify the choice of distributions we will use during the inference process.

${ }^{3}$ Such channel can e.g. be established via a sub-6 GHz link in a nonstand-alone deployment. Control feedback channel design details will not be discussed here due to space constraints.
}

channel gain $\alpha$ as well as the noise variance $\sigma^{2}$ is necessary to make such a search strategy usable in practice. We then detail our main contribution, which consists of augmenting HiePM with a novel variational model comparison based approximate inference framework [10] to account for the uncertainties about $\alpha$ and $\sigma_{B}^{2}$ and thus overcome the shortcomings, as detailed in the introduction, of the vanilla HiePM and modified HiePM schemes proposed in [7] and [9] respectively.

\section{A. Sequential Active Learning via the HiePM Strategy}

We illustrate here the use of vanilla HiePM scheme [7] for device $A$ (an analogous strategy will be used for device $B)$. HiePM selects $\boldsymbol{f}_{A, t+1}$ based on the posterior at time $t$ of the incidence angle $\phi_{A}$. We discretize ${ }^{4}$ the noisy beam search problem above by assuming that the beam search resolution $\delta_{A}{ }^{5}$ is an integer power of two and that the AoA $\phi_{A}$ is of the form:

$$
\phi_{A} \in\left\{\phi_{A, 1}, \ldots, \phi_{A, \delta_{A}}\right\}, \phi_{A, i}=\theta_{A, 1}+\frac{(i-1)}{\delta_{A}}\left(\theta_{A, 2}-\theta_{A, 1}\right)
$$

With the above setup, the posterior distribution of $\phi_{A}$ given all measurements up to time $t$ (collected in vector $\boldsymbol{y}_{B, 1: t}$ ), can be written as a $\delta_{A}$-dimensional vector $\pi_{A}(t)$ with entries

$$
\pi_{A, i}(t):=\operatorname{Pr}\left(\phi_{A}=\phi_{A, i} \mid \boldsymbol{y}_{B, 1: t}\right), \quad i=1, \ldots, \delta_{A} .
$$

The posterior probability of $\phi_{A}$ being in a certain range, say $\mathcal{R}_{i}^{m}$, can be computed as

$$
\pi_{A, \mathcal{R}_{i}^{m}}(t):=\sum_{\phi_{A, i} \in \mathcal{R}_{i}^{m}} \pi_{A, i}(t) .
$$

The HiePM strategy examines the posterior probability $\pi_{A, R_{i}^{m}}(t)$ for all $i=1, \ldots, S_{A}$ and $m=1, \ldots, 2^{i}$ and selects $f_{A, t+1} \in \mathcal{C}_{S}$ to be the beamformer corresponding to the angular range that satisfies:

$$
\left(i_{t+1}^{*}, m_{t+1}^{*}\right)=\underset{(i, m)}{\arg \min }\left|\pi_{A, \mathcal{R}_{i}^{m}}(t)-\frac{1}{2}\right|
$$

Doing so, it is guaranteed [7] to sequentially refine the width of the beamformer around the true incidence angle $\phi_{A}$.

Next we describe how the posterior bielief around $\phi_{A}$ is updated once a new measurement is taken with the pair of beamformers chosen previously with HiePM. Based on the measurement model in (2), the posterior update at time instant $t+1$ can be expressed using Bayes rule as

$$
\begin{gathered}
\pi_{A, i}(t+1) \propto \pi_{A, i}(t) f\left(y_{B, t+1} \mid \phi_{A}=\phi_{A, i}\right), \\
i=1, \ldots, \delta_{A}
\end{gathered}
$$

where $f\left(y_{B, t+1} \mid \phi_{A}=\phi_{A, i}\right)$ is the likelihood of $\phi_{A}$ from measurement $y_{B, t+1}$. Unfortunately, the likelihood term above cannot be calculated in closed form due to the unknown channel gain $\alpha$, noise variance $\sigma_{B}^{2}$ and incidence angle $\phi_{B}$.

We will show next, how "V-HiePM" is able, using the variational model based approximate inference framework described in [10], to infer all above unknowns and uses them

\footnotetext{
${ }^{4}$ Such discretization approaches the original problem of initial access as $\delta_{A} \rightarrow 0$ [7].

${ }^{5}$ To support this level of resolution, the corresponding number of levels of the hierarchical beamforming codebook at device $A$ should be : $S_{A}=$ $\log _{2}\left(\delta_{A}\right)$.
} 
efficiently to calculate the posterior update needed for HiePM, in a consistent and elegant way ${ }^{6}$.

\section{B. The V-HiePM Scheme}

We explain first the variational model based approximate inference framework used in its most general form, then show how to apply it to our problem to derive posterior updates for our parameters of interest.

1) Variational Model Comparison based Posterior Update: We start by listing the different types of variables that the variational model comparison based approximate inference framework deals with:

- $\boldsymbol{X}$ is the observed data vector, in our case is $\boldsymbol{y}_{B, 1: t+1}$.

- $\boldsymbol{Z}=\left(\boldsymbol{Z}_{1}, \boldsymbol{Z}_{2}, \ldots, \boldsymbol{Z}_{L}\right)$ denotes the $L$-dim vector of latent variables that parameterize the measurement model (2). In our case, $\boldsymbol{Z}=\left(\alpha, \sigma_{B}^{2}\right)$.

- $m \in\left\{1,2, \ldots, \delta_{A} \times \delta_{B}\right\}$ denotes the $m$ th pair of angles $\left(\phi_{A, i_{m}}, \phi_{B, j_{m}}\right)$, with $i_{m} \in\left\{1, \ldots, \delta_{A}\right\}$, and $j_{m} \in\left\{1, \ldots, \delta_{B}\right\}$. Choosing a certain label $m$ is equivalent to assuming that our measurement model in (2) is parameterized by the the $m$ th pair of angles.

The framework performs joint inference on the hidden variables to find a set of distributions $\{q(\boldsymbol{Z} \mid m), q(m)\}_{1: m}$ that approximate the true posterior $p(\boldsymbol{Z}, m \mid \boldsymbol{X})$, by minimizing the Kullback-Leibler (KL) divergence:

$$
K L(q(\boldsymbol{Z} \mid m) q(m), p(\boldsymbol{Z}, m \mid \boldsymbol{X})) .
$$

HiePM then uses the approximate incidence angle posterior $q(m)$ to decide which is the best measurement model candidate fitting the observed data vector $\boldsymbol{X}$. Algorithm 1 lists the steps required to perform such operations. We omit the mathematical derivation because of space constrains and refer to [10, Chapter 10.4$]$ for such details.

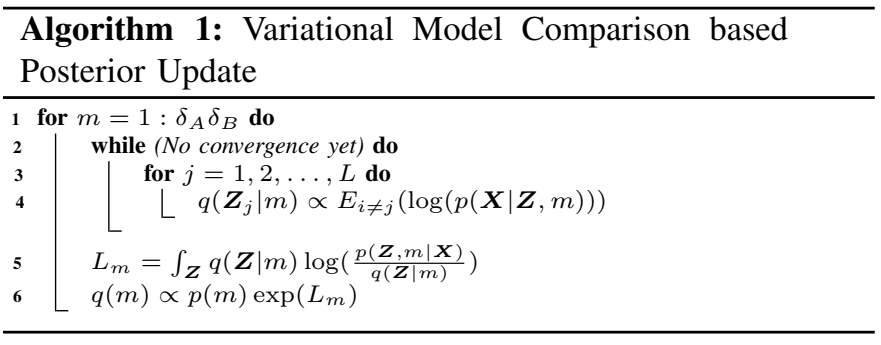

2) Posterior Update for our measurement Model and the overall V-HiePM Algorithm: From our measurement model (2), we have

$$
p(\boldsymbol{X}, \boldsymbol{Z}, m)=p\left(y_{B, 1: t} \mid \alpha, \nu_{B}, m\right) p(\alpha) p\left(\nu_{B}\right) p(m)
$$

where $\nu_{B}=\sigma_{B}^{-2}$ is the noise precision at device $B ; p(\boldsymbol{X} \mid \boldsymbol{Z}, m)=p\left(y_{B, 1: t} \mid \alpha, \nu_{B}, m\right)=$ $\prod_{i=1}^{t+1} C N\left(\boldsymbol{y}_{B, i} ; \sqrt{P} \alpha \boldsymbol{w}_{B, i}^{\mathrm{H}} \boldsymbol{A}_{m} \boldsymbol{f}_{A, i}, \sigma_{B}^{2}\right)$ is the likelihood

\footnotetext{
${ }^{6}$ As it will be detailed below, such an inference framework lends itself naturally in the HiePM context: we make the best use of the measurements by first estimating posteriors over the channel gain and noise variance and then use those to robustly update the angle of incidence posterior, doing so allows V-HiePM to take the channel's gain and noise variance estimation uncertainties properly into account when deriving the posterior of the incidence angles, thus making a robust HiePM based decision when choosing the next precoder/combiner pair to use.
}

of our measurement model (we assume here that the sequential noise samples are i.i.d); $p(\alpha)=C N\left(\alpha ; \alpha_{0}, \beta_{0}\right)$ is the prior belief over $\alpha$, considered to be Gaussian with a known initial mean $\alpha_{0}$ and initial precision $\beta_{0}{ }^{7}$; $p\left(\nu_{B}\right)=\Gamma\left(\nu_{B} ; a_{0}, b_{0}\right)$ is the non informative prior belief over $\nu_{B}$, with parameters $a_{0}=0$ and $b_{0}=0$; finally, $p(m)=\frac{1}{\delta_{A} \delta_{B}}$ is the prior belief over $m$, which is assumed to be uniform to make it non informative as well ${ }^{8}$. In addition, $\boldsymbol{A}_{m}=\boldsymbol{a}_{B}\left(\phi_{B, j_{m}}\right) \boldsymbol{a}_{A}^{\mathrm{H}}\left(\phi_{A, i_{m}}\right)$ is the assumed unfaded channel matrix under the $m$ th pair of incidence angles.

The obtained approximate posteriors for $\alpha$ and $\nu_{B}$, up to the measurement iteration $t$, can be shown to keep the form of their respective priors, but with parameters that depend on the measurement vector $\boldsymbol{y}_{B, 1: t}: q_{t}(\alpha \mid m)$ has the form of complex Gaussian pdf with mean $\alpha_{t, m}$ and precision $\beta_{t, m}$ reading

$$
\begin{gathered}
\beta_{t, m}=\frac{a_{t, m}}{b_{t, m}} \sum_{d=1}^{t}\left|\sqrt{P} \boldsymbol{w}_{B, t}^{\mathrm{H}} \boldsymbol{A}_{m} \boldsymbol{f}_{A, t}\right|^{2}+\beta_{0} \\
\alpha_{t, m}=\frac{a_{t, m}}{b_{t, m} \beta_{t, m}} \sum_{d=1}^{t}\left(\sqrt{P} \boldsymbol{w}_{B, t}^{\mathrm{H}} \boldsymbol{A}_{m} \boldsymbol{f}_{A, t}\right)^{*} y_{B, d}+\frac{\alpha_{0} \beta_{0}}{\beta_{t, m}}
\end{gathered}
$$

and $q_{t}\left(\nu_{B} \mid m\right)^{9}$ follows a Gamma pdf with parameters shape and rate parameters $a_{t, m}$ and $b_{t, m}$ given by

$$
\begin{gathered}
a_{t, m}=a_{0}+t, \\
b_{t, m}=b_{0}-2 R e\left(\sum_{d=1}^{t}\left(\sqrt{P} \boldsymbol{w}_{B, t}^{\mathrm{H}} \boldsymbol{A}_{m} \boldsymbol{f}_{A, t}\right)^{*} y_{B, d} \alpha_{t, m}^{*}\right)+ \\
\sum_{d=1}^{t}\left[\left|y_{B, d}\right|^{2}+\left(\frac{1}{\beta_{t, m}}+\left|\alpha_{t, m}\right|^{2}\right)\left|\sqrt{P} \boldsymbol{w}_{B, t}^{\mathrm{H}} \boldsymbol{A}_{m} \boldsymbol{f}_{A, t}\right|^{2}\right]
\end{gathered}
$$

Note that the choice of our prior distributions is not arbitrary, the priors chosen above correspond to the maximum entropy distributions [11] that respect constraints that need to be put on their respective parameters, namely $\alpha$ being a complex variable having a known initial mean and variance, $\nu_{B}$ being a non negative variable and $m$ being a discrete variable). Such a choice makes our proposal assume the least information about our measurement model's unknowns.

The posterior of the model, indexed by $m$, is then updated following Lines 5 and 6 in Algorithm 1, where $L_{m}$ reads

$$
\begin{aligned}
L_{t, m} & =\log \left(\frac{1}{\beta_{t, m}^{2}}\right)+a_{t, m}\left(1-\log \left(b_{t, m}\right)\right)+\log \left(\Gamma\left(a_{t, m}\right)\right) \\
& -b_{0} \frac{a_{t, m}}{b_{t, m}}-\left(\sum_{d=1}^{t}\left|y_{B, d}\right|^{2} \frac{a_{t, m}}{b_{t, m}}-\beta_{t, m}\left|\alpha_{t, m}\right|^{2}\right)
\end{aligned}
$$

\footnotetext{
${ }^{7}$ The first and second order moments of $\alpha$ are the only assumed known values in our model.

${ }^{8}$ In the above, $C N(\cdot ; \mu, \lambda)$ denotes the complex Gaussian pdf with mean $\mu$ and precision $\lambda, \Gamma(\cdot ; a, b)$ denotes the Gamma pdf with shape and rate parameters $a$ and $b$.

${ }^{9}$ Note that (10) and (11) can be re-written, after performing some algebra, in a recursive format w.r.t their terms involving summation over measurements epochs. This results in a significant reduction of the algorithm's memory and computation complexity footprint.
} 
The posteriors over $\phi_{A, i_{m}}$ and $\phi_{B, j_{m}}$ are obtained from the posterior $q_{t}(m)$ as

$$
\begin{array}{r}
q_{A, t}(i)=\sum_{\left\{m: i_{m}=i\right\}} q_{t}(m), \quad i=1, \ldots, \delta_{A} \\
q_{B, t}(j)=\sum_{\left\{m: j_{m}=j\right\}} q_{t}(m), \quad j=1, \ldots, \delta_{B}
\end{array}
$$

The posterior probability of the incidence angles $\phi_{A}$ and $\phi_{B}$ to be in a certain range $\mathcal{R}_{A, i}^{n}$ and $\mathcal{R}_{B, j}^{p}$ resp, read as:

$$
\begin{aligned}
q_{A, t}\left(\mathcal{R}_{A, i}^{n}\right) & :=\sum_{\phi_{A, i} \in \mathcal{R}_{A, i}^{n}} q_{A, t}(i), \\
q_{B, t}\left(\mathcal{R}_{B, j}^{p}\right) & :=\sum_{\phi_{B, j} \in \mathcal{R}_{B, j}^{p}} q_{B, t}(i),
\end{aligned}
$$

The vanilla HierPM scheme is then applied separately to $q_{A, t}(i)$ and $q_{B, t}(j)$, to choose the pair of beamformers to use for the next measurement occasion.

The modes $\hat{\alpha}_{t}$ and $\hat{\nu_{B t}}$ of the approximate posteriors $q_{t}\left(\alpha \mid m_{t}^{*}\right)$ and $q_{t}\left(\nu_{B} \mid m_{t}^{*}\right)$, with $m_{t}^{*}=\arg \max _{m}\left(q_{t}(m)\right)$, can be seen as approximations of the MMSE estimates of $\alpha$ and $\nu_{B}$ respectively. These estimates are given by:

$$
\hat{\alpha}_{t}=\alpha_{t, m_{t}^{*}}, \quad \hat{\nu_{B} t}=a_{t, m_{t}^{*}} / b_{t, m_{t}^{*}} .
$$

Algorithm 2 runs all above operations in a loop, until the measurement budget is exhausted: device $B$ decides which pair of beamformers devices $A$ and $B$ shall use to take the next measurement by applying the HiePM scheme separately to the current posteriors $q_{A, t}$ and $q_{B, t}$, it then takes a new measurement $y_{B, t+1}$ with those latter, and finally run variational inference to derive approximate posteriors of $\nu_{B}=\frac{1}{\sigma_{B}^{2}}$, $\alpha$ as well as of $\phi_{A}$ and $\phi_{B}$.

\section{NumericAl Results}

To assess the effectiveness of V-HiePM, we run Monte Carlo simulations on a setup with two hybrid digital-analog beamforming devices $A$ and $B$. The channel matrix $\boldsymbol{H} \in \mathbb{C}^{N_{B} \times N_{A}}$ reads

$$
\boldsymbol{H}=\alpha \boldsymbol{a}_{B}\left(\phi_{B}\right) \boldsymbol{a}_{A}^{\mathrm{H}}\left(\phi_{A}\right)+\sum_{l=1}^{L} \alpha_{l} \boldsymbol{a}_{B}\left(\phi_{B, l}\right) \boldsymbol{a}_{A}^{\mathrm{H}}\left(\phi_{A, l}\right)
$$

and contains one dominant multipath component and $L$ scattered components. All incidence angles are independently drawn from a uniform distribution between 0 and $\pi$. The channel gains are independently drawn from a a set of complex Gaussian distribution with mean 0 and variances fulfilling $\operatorname{Var}\{\alpha\}+\sum_{l} \operatorname{Var}\left\{\alpha_{l}\right\}=1$, so that the average SNR $\rho$ between the $n$th element of the array at $A$ and the $m$ th element of the array at $B$ equals $\mathbb{E}\left\{\left|H_{n m}\right|^{2}\right\} / \mathbb{E}\left\{\left|\sigma_{B}\right|^{2}\right\}=1 / \sigma_{B}^{2}{ }^{10}$. In all simulations below, the two devices are equipped with identical arrays made of $N_{A}=N_{B}=32$ elements, digitally controlled with $N_{A}^{R F}=N_{B}^{R F}=8 \mathrm{RF}$ chains. Device $A$ uses a codebook $\mathcal{C}^{A}$ with a depth of $S_{A}=\log _{2}\left(\delta_{A}\right), \delta_{A}=128 . \mathcal{C}^{A}$ is built

\footnotetext{
${ }^{10} H_{n m}$ is the channel coefficient between device $B$ 's $n$th array element and device $A$ 's $m$ th array element, and $\mathbb{E}\{\}$ is the expectation operator.
}

using the orthogonal matching pursuit as described in [4]. A similar codebook, $\mathcal{C}^{B}$, is used for device $B .{ }^{11}$

We benchmark our algorithm's beamforming gain after $t$ measurements, defined as:

$$
\boldsymbol{G}_{\boldsymbol{v} \boldsymbol{h}}=\left|\boldsymbol{w}^{\mathrm{H}}\left(\phi_{B, \hat{k}_{t, B}}\right) \boldsymbol{H} \boldsymbol{f}\left(\phi_{A, \hat{k}_{t, A}}\right)\right|^{2}
$$

with different measurement budget sizes and under different channel assumptions (note the the exhaustive search needs $N_{A} N_{B}=16384$ measurements to settle), against that of the different state of the art schemes listed below:

- $G_{p h}$ of the vanilla HiePM scheme of [7]. Here, such a scheme assumes that most of the energy in the channel is concentrated in the path corresponding to the known gain $\alpha$ and all other gains $\alpha_{l}$ are null, it also assumes that $\sigma_{B}^{2}$ is known. In such case, the posterior update is done, simply using Bayes rule as in equation (21) in [7], on the beam pair corresponding to that main path, and then HiePM is applied to the marginals over those angles separately, similar to what V-HiePM does.

- $G_{b s}$ of the noisy binary search algorithm of [4], which is achieved by $4 \log _{2}\left(\max \left\{N_{A}, N_{B}\right\}\right)=28$ measurements.

As a reference, we consider as well the best achievable beamforming gain of the codebook, defined as

$$
\boldsymbol{G}_{\boldsymbol{m a x}}=\max _{\left\{\boldsymbol{w} \in \mathcal{C}_{S_{B}}^{B}, \boldsymbol{f} \in \mathcal{C}_{S_{A}}^{A}\right\}}\left|\boldsymbol{w}^{\mathrm{H}} \boldsymbol{H} \boldsymbol{f}\right|^{2} .
$$

\footnotetext{
${ }^{11}$ Note that the multi-RF chain setups are used solely to help build acceptable RF codebooks [4], and are not used for multi-stream MIMO operations.
}

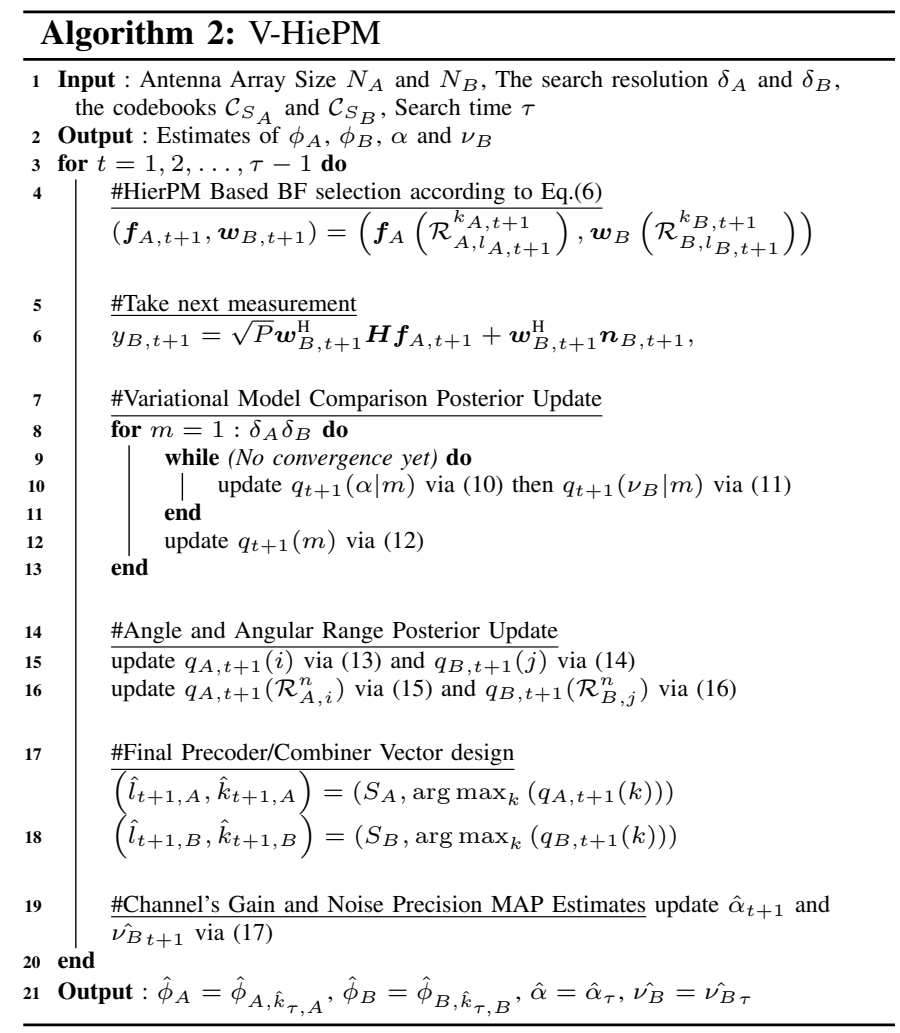




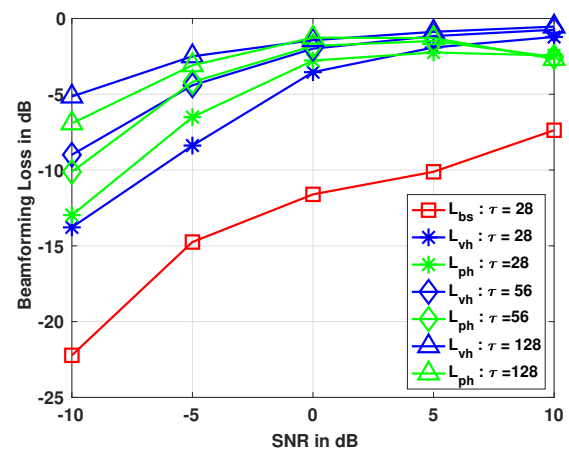

Figure 1: Beamforming loss of the different search schemes in a channel with $L=0$ scattered components.

We begin by assuming that only the dominant component is present (i.e. $L=0$ ). Fig. 1 shows the beamforming losses of the benchmarked algorithms with respect to the optimum pair of beamformers, defined as $\boldsymbol{L}_{\boldsymbol{v} \boldsymbol{h}}=\boldsymbol{G}_{\boldsymbol{v} \boldsymbol{h}} / \boldsymbol{G}_{\boldsymbol{m a x}}, \boldsymbol{L}_{\boldsymbol{p h}}=$ $\boldsymbol{G}_{p \boldsymbol{h}} / \boldsymbol{G}_{\boldsymbol{m a x}}$, and $\boldsymbol{L}_{\boldsymbol{b s}}=\boldsymbol{G}_{\boldsymbol{b s}} / \boldsymbol{G}_{\max }$. The results show the superiority of our scheme compared to the binary search of [4], and that it achieves similar or even better performance compared to vanilla HiePM with perfect CSI and operating SNR knowledge. It can be observed that the vanilla HiePM scheme with perfect channel gain knowledge saturates at high SNR: this is an effect of the algorithm assuming that the component's incidence angle lies on a discrete grid of values, whereas the actual angles are sampled from a continuous distribution. Our proposed method is less sensitive to this model mismatch, due to the estimation of the channel gain and inverse noise variance: in practice, these estimates partly account for the mismatch in the assumed values of the angles and provide robustness to the overall procedure.

Next, we explore the robustness of the proposed method against channels containing more than one multipath component. For this, we consider a channel with $L=3$ scattered components with gains of equal variance, and with the power ratio between the dominant and scattered components being $L O S_{R}=\mathbb{E}\left\{\alpha^{2}\right\} /\left(\mathbb{E}\left\{\alpha^{2}\right\}+\sum_{l} \mathbb{E}\left\{\alpha_{l}^{2}\right\}\right)$. Fig. 2 shows beamforming gains achieved by our algorithm after 100 measurements compared to the maximum gains achievable $\boldsymbol{G}_{\boldsymbol{m a x}}$.

As it can be observed, the maximum achievable beamforming gain decreases as the power is more evenly distributed among the channel's components. Although V-HiePM assumes the existence of a single component, it shows remarkable resilience to the presence of other components. Even when all components in the model have comparable power, our proposed method is able to perform within $2 \mathrm{~dB}$ of the optimum for sufficiently high SNR.

\section{CONCLUSiOn AND Future WORK}

We proposed in this work a variational Bayesian online learning scheme that enables initial access for hybrid digitalanalog enabled devices operating in mmWave wireless channels. When compared to state of the art beam acquisition schemes, our method shows superiority, in terms of balancing

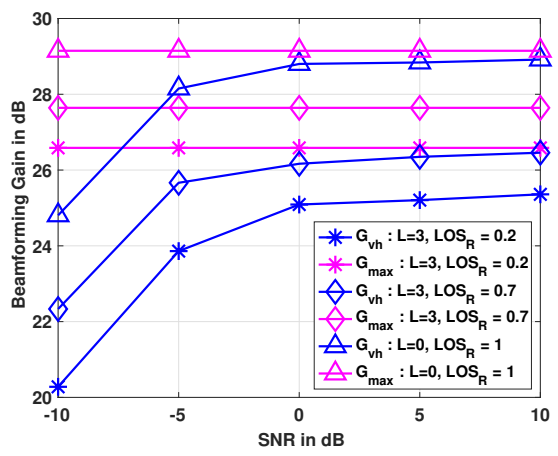

Figure 2: V-HiePM performance in channels with different power ratios between the dominant path and $L=3$ scattered components.

the beam search time versus achieving higher beamforming gain, in being able to properly do so while accounting for uncertainties on the unknown CSI (gain and noise variance) and in being very resilient to the dominant single path assumption. Even though the scheme is derived based on a discretized model of the angles of incidence of the channel's main component, it showed great robustness against off-grid angles as well as working with a realistic codebook implementation. Further research will focus on adapting the proposed online learning algorithm to operating in time-varying channels.

\section{REFERENCES}

[1] R. W. Heath, N. González-Prelcic, S. Rangan, W. Roh, and A. M. Sayeed, "An overview of signal processing techniques for millimeter wave MIMO systems," IEEE Journal of Selected Topics in Signal Processing, vol. 10, no. 3, pp. 436-453, Apr. 2016.

[2] S. Hur, S. Baek, B. Kim, Y. Chang, A. F. Molisch, T. S. Rappaport, K. Haneda, and J. Park, "Proposal on millimeter-wave channel modeling for 5G cellular systems," IEEE Journal of Selected Topics in Signal Processing, vol. 10, no. 3, pp. 454-469, Apr. 2016.

[3] O. E. Ayach, S. Rajagopal, S. Abu-Surra, Z. Pi, and R. W. Heath, "Spatially sparse precoding in millimeter wave MIMO systems," IEEE Transactions on Wireless Communications, vol. 13, no. 3, pp. 14991513, Mar. 2014.

[4] A. Alkhateeb, O. E. Ayach, G. Leus, and R. W. Heath, "Channel estimation and hybrid precoding for millimeter wave cellular systems," IEEE Journal of Selected Topics in Signal Processing, vol. 8, no. 5, pp. 831-846, Oct. 2014.

[5] N. Akdim, C. N. Manchón, M. Benjillali, and E. de Carvalho, "Ping pong beam training for multi stream MIMO communications with hybrid antenna arrays," in 2018 IEEE Globecom Workshops (GC Wkshps), Dec. 2018, pp. 1-7.

[6] Y. Ding, S. Chiu, and B. D. Rao, "Bayesian channel estimation algorithms for massive MIMO systems with hybrid analog-digital processing and low-resolution ADCs," IEEE Journal of Selected Topics in Signal Processing, vol. 12, no. 3, pp. 499-513, Jun. 2018.

[7] S. Chiu, N. Ronquillo, and T. Javidi, "Active learning and CSI acquisition for mmwave initial alignment," IEEE Journal on Selected Areas in Communications, pp. 1-1, 2019.

[8] O. Shayevitz and M. Feder, "Optimal feedback communication via posterior matching," IEEE Transactions on Information Theory, vol. 57, no. 3, pp. 1186-1222, Mar. 2011.

[9] N. Ronquillo, S. Chiu, and T. Javidi, "Sequential learning of CSI for mmwave initial alignment," CoRR, vol. abs/1912.12738, 2019. [Online]. Available: http://arxiv.org/abs/1912.12738

[10] C. M. Bishop, Pattern Recognition and Machine Learning (Information Science and Statistics). Berlin, Heidelberg: Springer-Verlag, 2006.

[11] M. U. Thomas, "A generalized maximum entropy principle," Operations Research, vol. 27, no. 6, pp. 1188-1196, 1979. 